\begin{tabular}{|c|l|}
\hline Title & Global existence of small amplitude solutions for the Klein-Gordon-Zakharov equations \\
\hline Author(s) & Tsutaya, K. \\
\hline Citation & Hokkaido University Preprint Series in Mathematics, 273, 1-11 \\
\hline Issue Date & 199412-1 \\
\hline DOI & 10.14943/83420 \\
\hline Doc URL & http://hdl.handle.net/2115/69024 \\
\hline Type & bulletin (article) \\
\hline File Information & pre273.pdf \\
\hline
\end{tabular}

Instructions for use 
Global Existence of Small Amplitude Solutions for the Klein-Gordon-Zakharov Equations

Kimitoshi Tsutaya

Series $\sharp 273$. December 1994 


\section{HOKKAIDO UNIVERSITY PREPRINT SERIES IN MATHEMATICS}

$\sharp 247$ N. Hayashi, K. Kato and T. Ozawa, Dilation Method and smoothing Effect of Solutions to the Benjamin-ono Equation, 17 pages. 1994.

$\sharp 248$ H. Kikuchi, Sheaf cohomology theory for measurable spaces, 12 pages. 1994.

$\sharp 249$ A. Inoue, Tauberian theorems for Fourier cosine transforms, 9 pages. 1994.

$\sharp 250$ S. Izumiya, G. T. Kossioris, Singularities for viscosity solutions of Hamilton-Jacobi equations, 23 pages. 1994.

\#251 H. Kubo, K. Kubota, Asymptotic behaviors of radially symmetric solutions of $\square u=|u|^{p}$ for super critical values $p$ in odd space dimensions, 51 pages. 1994.

\#252 T. Mikami, Large Deviations and Central Limit Theorems for Eyrand-Farlie-Gumbel-Morgenstern Processes, 9 pages. 1994.

\#253 T. Nishimori, Some remarks in a qualitative theory of similarity pseudogroups, 19 pages. 1994.

$\sharp 254$ T. Suwa, Residues of complex analytic foliations relative to singular invariant subvarieties, 15 pages. 1994.

$\sharp 255$ T. Tsujishita, On Triple Mutual Information, 7 pages. 1994.

\#256 T. Tsujishita, Construction of Universal Modal World based on Hyperset Theory, 15 pages. 1994.

$\sharp 257$ A. Arai, Trace Formulas, a Golden-Thompson Inequality and Classical Limit in Boson Fock Space, 35 pages. 1994.

\#258 Y-G. Chen, Y. Giga, T. Hitaka and M. Honma, A Stable Difference Scheme for Computing Motion of Level Surfaces by the Mean Curvature, 18 pages. 1994.

\#259 K. Iwata, J. Schäfer, Markov property and cokernels of local operators, 7 pages. 1994.

$\sharp 260$ T. Mikami, Copula fields and its applications, 14 pages. 1994.

$\sharp 261$ A. Inoue, An Abel-Tauber theorem for Fourier sine transforms, 6 pages. 1994.

$\sharp 262$ N. Kawazumi, Homology of hyperelliptic mapping class groups for surfaces, 13 pages. 1994.

$\sharp 263$ Y. Giga, M. E. Gurtin, A comparison theorem for crystalline evolution in the plane, 14 pages. 1994.

$\sharp 264$ J. Wierzbicki, On Commutativity of Diagrams of Type $\mathrm{II}_{1}$ Factors, 26 pages. 1994.

$\sharp 265$ N. Hayashi, T. Ozawa, Schrödinger Equations with nonlinearity of integral type, 12 pages. 1994.

$\sharp 266$ T. Ozawa, On the resonance equations of long and short waves, 8 pages. 1994.

$\sharp 267$ T. Mikami, A sufficient condition for the uniqueness of solutions to a class of integro-differential equations, 9 pages. 1994.

$\sharp 268$ Y. Giga, Evolving curves with boundary conditions, 10 pages. 1994.

$\sharp 269$ A. Arai, Operator-theoretical analysis of representation of a supersymmetry algebra in Hilbert space, 12 pages. 1994.

$\$ 270$ A. Arai, Gauge theory on a non-simply-connected domain and representations of canonical commutation relations, 18 pages. 1994.

$\sharp 271$ S. Jimbo, Y. Morita and J. Zhai, Ginzburg landau equation and stable steady state solutions in a non-trivial domain, 17 pages. 1994.

$\sharp 272$ S. Izumiya, A. Takiyama, A time-like surface in Minkowski 3-space which contains light-like lines, 7 pages. 1994. 


\title{
Global Existence of Small Amplitude Solutions for the Klein-Gordon-Zakharov Equations
}

\author{
Kimitoshi Tsutaya * \\ Department of Mathematics \\ Hokkaido University \\ Sapporo 060, Japan
}

Key words and phrases: Global existence, Klein-Gordon-Zakharov equations.

\section{Introduction}

We consider the Cauchy problem of the Klein-Gordon-Zakharov equations in three space dimensions :

$$
\begin{aligned}
& \partial_{t}^{2} u-\Delta u+u=-n u, \quad t>0, \quad x \in \mathbf{R}^{3}, \\
& \partial_{t}^{2} n-\Delta n=\Delta|u|^{2}, \quad t>0, \quad x \in \mathbf{R}^{3}, \\
& u(0, x)=u_{0}(x), \quad \partial_{t} u(0, x)=u_{1}(x), \\
& n(0, x)=n_{0}(x), \quad \partial_{t} n(0, x)=n_{1}(x),
\end{aligned}
$$

where $\partial_{t}=\partial / \partial t$, and $u(t, x)$ and $n(t, x)$ are functions from $\mathbf{R}_{+} \times \mathbf{R}^{3}$ to $\mathbf{C}^{3}$ and from $\mathbf{R}_{+} \times \mathbf{R}^{3}$ to $\mathbf{R}$, respectively. The system (1.1)-(1.2) describes the propagation of strong turbulence of the Langmuir wave in a high frequency plasma (see [14]).

Many results have been obtained concerning the global existence of small amplitude solutions for the coupled systems of the Klein-Gordon and wave equations with quadratic nonlinearity (see, e.g., $[1-4,6,7,9-11])$. Two methods are known to be applicable to solve those systems. One is to use the invariant Sobolev space with respect to the generators of the Lorentz group. This was developed by Klainerman [7]. He also introduced the notion of the null condition to prove the existence of global solutions for the wave equations with quadratic nonlinearity. We note that

*Partially supported by THE SUHARA MEMORIAL FOUNDATION. 
the null condition technique is based on the Lorentz invariance of the equations. Recently, Bachelot [1] and Georgiev [3] improved the null condition technique to show the global existence result for the Dirac-Klein-Gordon equations and the MaxwellDirac equations, respectively, which are physically important (see also Georgiev [4]). Another method is based on the theory of normal forms introduced by Shatah [10], which is an extension of Poincaré's theory of normal forms for the ordinary differential equations to the partial differential equations. The idea of this method is to transform the original system with quadratic nonlinearity into a new system with cubic nonlinearity. See also [12] and its references. Recently, applying the argument of normal forms to (1.1)-(1.2), Ozawa, Tsutaya and Tsutsumi [9] have proved the existence of global solutions to (1.1)-(1.3) for small initial data. However, in [9] one needs the high regularity assumptions on the data to ensure the global existence. Moreover, the global solution $n$ of (1.1)-(1.3) constructed by [9] must belong to the homogeneous Sobolev space $\dot{H}^{-1}$ of negative index. In this paper we prove that there exist the global solutions of (1.1)-(1.3) for small data using the invariant Sobolev space but without applying the null condition technique. We improve the regularity requirements on the intial data. Our proof seems simpler and shorter than that of $[9]$.

Before we state the main results in the present paper, we give several notations. We put $\partial_{j}=\partial / \partial x_{j}$ for $j=1,2,3$. Let $\Gamma=\left(\Gamma_{j} ; j=1, \cdots, 10\right)$ denote the generators of the Poincaré group $\left(\partial_{t}, \partial_{1}, \partial_{2}, \partial_{3}, L_{1}, L_{2}, L_{3}, \Omega_{12}, \Omega_{23}, \Omega_{13}\right)$, where

$$
\begin{aligned}
& L_{j}=x_{j} \partial_{t}+t \partial_{j}, \quad j=1,2,3 \\
& \Omega_{i j}=x_{i} \partial_{j}-x_{j} \partial_{i}, 1 \leq i<j \leq 3,
\end{aligned}
$$

and we put

$$
\partial=\left(\partial_{t}, \partial_{1}, \partial_{2}, \partial_{3}\right)
$$

For a multi-index $\alpha=\left(\alpha_{1}, \alpha_{2}, \alpha_{3}\right)$, we put

$$
\partial_{x}^{\alpha}=\partial_{1}^{\alpha_{1}} \partial_{2}^{\alpha_{2}} \partial_{3}^{\alpha_{3}}
$$


For a multi-index $\alpha=\left(\alpha_{1}, \alpha_{2}, \alpha_{3}, \alpha_{4}\right)$, we put

$$
\partial^{\alpha}=\partial_{t}^{\alpha_{1}} \partial_{1}^{\alpha_{2}} \partial_{2}^{\alpha_{3}} \partial_{3}^{\alpha_{4}}
$$

For a multi-index $\alpha=\left(\alpha_{1}, \cdots, \alpha_{10}\right)$, we put

$$
\Gamma^{\alpha}=\Gamma_{1}^{\alpha_{1}} \cdots \Gamma_{10}^{\alpha_{10}}
$$

For $1 \leq p \leq \infty$, let $L^{p}$ denote the standard $L^{p}$ space on $\mathbf{R}^{3}$. For $m \geq 0$ and $s \geq 0$, we define the weighted Sobolev space $H^{m, s}$ on $\mathbf{R}^{3}$ as follows:

$$
H^{m, s}=\left\{v \in L^{2} ;\left(1+|x|^{2}\right)^{s / 2}(1-\Delta)^{m / 2} v \in L^{2}\right\}
$$

We put $H^{m} \equiv H^{m, 0}$ for $m \geq 0$. Let $\omega=(1-\Delta)^{1 / 2}$ and $\omega_{0}=(-\Delta)^{1 / 2}$.

We have the following theorem concerning the global existence of solutions to (1.1)-(1.3) for small initial data.

Theorem $1.1 \quad$ Let $0<\varepsilon<1 / 6$ and $k \geq 4$. Assume that $u_{0} \in H^{k+5, k+4}, u_{1} \in$ $H^{k+4, k+4}, n_{0} \in H^{k+4, k+4}$ and $n_{1} \in H^{k+3, k+4}$. Then, there exists a $\delta>0$ such that if

$$
\left\|u_{0}\right\|_{H^{k+3, k+4}}+\left\|u_{1}\right\|_{H^{k+4, k+4}}+\left\|n_{0}\right\|_{H^{k+4, k+4}}+\left\|n_{1}\right\|_{H^{k+3, k+4}} \leq \delta
$$

then (1.1)-(1.3) has the unique global solutions $(u, n)$ satisfying

$$
\begin{aligned}
& u \in \bigcap_{j=0}^{k+4} C^{j}\left([0, \infty) ; H^{k+4-j}\right), \\
& n \in \bigcap_{j=0}^{k+4} C^{j}\left([0, \infty) ; H^{k+4-j}\right), \\
& \sum_{|\alpha|=k+4} \sup _{t \geq 0}(1+t)^{-\varepsilon}\left\{\left\|\partial_{t} \Gamma^{\alpha} u(t)\right\|_{L^{2}}+\left\|\omega \Gamma^{\alpha} u(t)\right\|_{L^{2}}\right\} \\
& +\sum_{|\alpha| \leq k+4} \sup _{t \geq 0}(1+t)^{-\varepsilon}\left\|\Gamma^{\alpha} u(t)\right\|_{L^{2}}+\sum_{|\alpha| \leq k+4} \sup _{t \geq 0}\left\|\Gamma^{\alpha} n(t)\right\|_{L^{2}} \\
& +\sum_{|\alpha| \leq k} \sup _{\substack{t \geq 0 \\
x \in \mathrm{R}^{2}}}\left\{\left|(1+t+|x|)^{3 / 2-2 \varepsilon} \Gamma^{\alpha} u(t, x)\right|\right. \\
& \left.+\left|(1+t+|x|) \Gamma^{\alpha} n(t, x)\right|\right\}<\infty
\end{aligned}
$$

Remark 1.1 (i) By (1.7), we see that the right hand sides of (1.1)-(1.2) are integrable in time and therefore we find that the solutions $(u, n)$ of $(1.1)-(1.3)$ 
constructed by Theorem 1.1 asymptotically approach the free solutions as $t \rightarrow \infty$. See [9].

(ii) As compared to [9], we have brought down the regularity assumptions on the data significantly. Instead, we need some spatial decay on the data, which is inevitable as far as the method depends on the invariant Sobolev norms.

(iii) The estimate (1.7) is close to the optimal one as regards the space-time behavior of solutions.

The following corollary follows easily from the proof of Theorem 1.1.

Corollary 1.2 In addition to all the assumptions in Theorem 1.1, if $u_{0}, u_{1}, n_{0}$, $n_{1} \in \bigcap_{m \geq 1} H^{m}$, then the solutions $(u, n)$ given by Theorem 1.1 satisfy

$$
u(t, x), n(t, x) \in C^{\infty}\left([0, \infty) \times \mathbf{R}^{3}\right) .
$$

We prove Theorem 1.1 by the contraction argument in the next section. The main tools in the proof are the decay estimate of the inhomogeneous linear KleinGordon equation due to Georgiev [4] and the Sobolev inequality in the Minkowski space by Klainerman [6, 8] and Hörmander [5]. We show the global existence result for (1.1)-(1.3) by using these two inequalities and adopting the weight function in the norm slightly different from [2,3] and [1]. Finally, we remark that the various constants in the estimate are simply denoted by $C$.

\section{Proof of Theorem 1.1.}

In this section we describe the proof of Theorem 1.1. We solve (1.1)-(1.3) by the contraction mapping principle. For $k \in \mathrm{N}, a \in \mathrm{R}$, we define the norms

$$
\begin{aligned}
|u|_{k, a} & =\sup _{\substack{s \geq 0 \\
y \in \mathbb{R}^{3}}}(1+s+|y|)^{-a} \sum_{|\alpha| \leq k}\left|\Gamma^{\alpha} u(s, y)\right|, \\
\|u\|_{k, a} & =\sup _{s \geq 0}(1+s)^{-a}\|u(s)\|_{k}
\end{aligned}
$$




$$
\begin{aligned}
\|u(t)\|_{k} & =\sum_{|\alpha| \leq k}\left\|\Gamma^{\alpha} u(t)\right\|, \\
\|u(t)\| & =\|u(t)\|_{L^{2}\left(\mathbf{R}^{3}\right)} .
\end{aligned}
$$

We put

$$
\begin{aligned}
X= & X(k, M) \\
= & \left\{(u(t), n(t)) ; u(t) \in \bigcap_{j=0}^{k+4} C^{j}\left([0, \infty) ; H^{k+4-j}\right),\right. \\
& n(t) \in \bigcap_{j=0}^{k+4} C^{j}\left([0, \infty) ; H^{k+4-j}\right), u(0, x)=u_{0}(x), \partial_{t} u(0, x)=u_{1}(x), \\
& \left.n(0, x)=n_{0}(x), \partial_{t} n(0, x)=n_{1}(x),\|(u, n)\|_{X} \leq M\right\},
\end{aligned}
$$

where

$$
\|(u, n)\|_{X}=|u|_{k,-3 / 2+2 \varepsilon}+\|u\|_{k+4, \varepsilon}+\|\partial u\|_{k+4, \varepsilon}+\|n\|_{k+4,0}(k \geq 4,0<\varepsilon<1 / 6),
$$

and $M$ is a positive constant, which will be determined later. We introduce the metric $d((u, n),(v, m))$ into $X$ as follows.

$$
d((u, n),(v, m))=\|(u-v, n-m)\|_{X}
$$

for $(u, n),(v, m) \in X$. We note that $X$ is complete with respect to the metric $d$. Given $(u, n) \in X$, we define the maps

$$
\begin{aligned}
& \chi_{1}:(u, n) \rightarrow \psi=\chi_{1}(u, n), \\
& \chi_{2}:(u, n) \rightarrow \varphi=\chi_{2}(u, n) \quad \text { for }(u, n) \in X
\end{aligned}
$$

by solving the Cauchy problem

$$
\begin{aligned}
& \partial_{t}^{2} \psi-\Delta \psi+\psi=-n u, \quad t>0, \quad x \in \mathbf{R}^{3}, \\
& \partial_{t}^{2} \varphi-\Delta \varphi=\Delta|u|^{2}, \quad t>0, \quad x \in \mathbf{R}^{3}, \\
& \psi(0, x)=u_{0}(x), \quad \partial_{t} \psi(0, x)=u_{1}(x), \\
& \varphi(0, x)=n_{0}(x), \quad \partial_{t} \psi(0, x)=n_{1}(x) .
\end{aligned}
$$


We put $\chi(u, n)=\left(\chi_{1}(u, n), \chi_{2}(u, n)\right)$. Let $\delta$ be a positive constant satisfying (1.4). We show that if $\delta$ is sufficiently small, then the map $\chi$ is a contraction from $X$ into itself.

We first evaluate $\psi=\chi_{1}(u, n)$. To derive the $L^{\infty}$ estimate of $\psi$, we use the following time decay estimate of solution of the linear Klein-Gordon equations due to Georgiev [4].

Lemma 2.1 Let $u(t, x)$ be a solution of the inhomogeneous linear KleinGordon equation :

$$
\left\{\begin{array}{lll}
\partial_{t}^{2} u-\Delta u+u=f, & t>0, & x \in \mathbf{R}^{3}, \\
u(0, x)=u_{0}(x), \quad \partial_{t} u(0, x)=u_{1}(x), & x \in \mathbf{R}^{3} .
\end{array}\right.
$$

Then, we have

$$
\begin{array}{r}
(1+t+|x|)^{3 / 2}|u(t, x)| \leq C \sum_{j=0}^{\infty} \sum_{|\alpha| \leq 4} \sup _{s \in(0, t)} \varphi_{j}(s)\left\|(1+s+|y|) \Gamma^{\alpha} f(s, y)\right\|_{L^{2}\left(\mathbf{R}_{y}^{3}\right)} \\
+C \sum_{j=0}^{\infty} \sum_{|\alpha| \leq 5}\left\|(1+|y|)^{3 / 2} \varphi_{j}(|y|) \Gamma^{\alpha} u(0, y)\right\|_{L^{2}\left(\mathbf{R}_{y}^{3}\right)}
\end{array}
$$

where $\left\{\varphi_{j}\right\}_{j=0}^{\infty}$ is a Littlewood-Paley partition of unity, i.e.,

$$
\begin{aligned}
& \sum_{j=0}^{\infty} \varphi_{j}(s)=1, \quad s \geq 0 ; \quad \varphi_{j} \in C_{0}^{\infty}(\mathbf{R}), \quad \varphi_{j} \geq 0 \quad \text { for all } j \geq 0 ; \\
& \operatorname{supp} \varphi_{j}=\left[2^{j-1}, 2^{j+1}\right] \quad \text { for } j \geq 1, \quad \operatorname{supp} \varphi_{0} \cap \overline{\mathbf{R}_{+}}=[0,2] .
\end{aligned}
$$

We apply $\Gamma^{\alpha}$ to $(2.1)$ and compute the equation of $\Gamma^{\alpha} \psi$ on the basis of the commutation relation

$$
[\square+1, \Gamma]=0
$$

Then, it follows from Lemma 2.1 and a finite dependence domain argument that

$$
\begin{aligned}
& (1+t+|x|)^{3 / 2-2 \varepsilon}\left|\Gamma^{\alpha} \psi(t, x)\right| \\
& \leq C \sum_{j=0}^{\infty} \sum_{|\lambda| \leq 4} \sup _{s \in(0, t)} \varphi_{j}(s)\left\|(1+s+|y|)^{1-2 \varepsilon} \Gamma^{\alpha+\lambda}(n u)(s, y)\right\| \\
& \quad+C \sum_{j=0}^{\infty} \sum_{|\lambda| \leq 5}\left\|(1+|y|)^{3 / 2} \varphi_{j}(|y|) \Gamma^{\alpha+\lambda} \psi(0, y)\right\| \\
& \quad+C \sum_{j=0}^{\infty} \sum_{|\lambda| \leq 4}\left\|(1+|y|)^{5 / 2} \varphi_{j}(|y|) \Gamma^{\alpha+\lambda} \partial_{t} \psi(0, y)\right\|
\end{aligned}
$$


for $|\alpha| \leq k$ (see [3], p. 867). From (2.1), we easily see that

$$
\begin{aligned}
& \sum_{j=0}^{\infty} \sum_{|\lambda| \leq 5}\left\|(1+|y|)^{3 / 2} \varphi_{j}(|y|) \Gamma^{\alpha+\lambda} \psi(0, y)\right\| \\
& +\sum_{j=0}^{\infty} \sum_{|\lambda| \leq 4}\left\|(1+|y|)^{5 / 2} \varphi_{j}(|y|) \Gamma^{\alpha+\lambda} \partial_{t} \psi(0, y)\right\| \leq C \delta, \quad|\alpha| \leq k .
\end{aligned}
$$

On the other hand, by the Sobolev inequality in the Minkowski space $\mathbf{R}^{3+1}$ due to Klainerman $[6,8]$ and Hörmander [5], we have

$$
\begin{aligned}
& \left\|(1+s+|y|)^{1-2 \varepsilon} \Gamma^{\alpha+\lambda}(n u)(s, y)\right\| \\
\leq & C \sum_{|\beta|+|\gamma|=|\alpha+\lambda|}\left\|(1+s+|y|)^{1-2 \varepsilon} \Gamma^{\beta} n \cdot \Gamma^{\gamma} u(s, y)\right\| \\
\leq & C(1+s)^{-\varepsilon}|n|_{[k / 2]+2,-1}\|u\|_{k+4, \varepsilon} \\
& +C(1+s)^{-1 / 2}\|n\|_{k+4,0}|u|_{[k / 2]+2,-3 / 2+2 \varepsilon} \\
\leq & C(1+s)^{-\varepsilon}\|n\|_{[k / 2]+5,0}\|u\|_{k+4, \varepsilon} \\
& \quad+C(1+s)^{-1 / 2}\|n\|_{k+4,0}|u|_{[k / 2]+2,-3 / 2+2 \varepsilon} \\
\leq & C(1+s)^{-\varepsilon} M^{2}, \quad|\alpha| \leq k,|\lambda| \leq 4 .
\end{aligned}
$$

Since

$$
\sum_{j=0}^{\infty} \sup _{s \in(0, t)} \varphi_{j}(s)(1+s)^{-\varepsilon} \leq C,
$$

inequalities (2.5)-(2.7) yield

$$
\sum_{|\alpha| \leq k}(1+t+|x|)^{3 / 2-2 \varepsilon}\left|\Gamma^{\alpha} \psi(t, x)\right| \leq C \delta+C M^{2}
$$

We next show the energy estimate of $\psi$. Applying $\Gamma^{\alpha}$ to (2.1) for $|\alpha| \leq k+4$, we have

$$
\begin{aligned}
& \left\|\Gamma^{\alpha} \psi(t)\right\|+\left\|\partial \Gamma^{\alpha} \psi(t)\right\| \\
\leq & C\left(\left\|u_{0}\right\|_{H^{k+5, k+4}}+\left\|u_{1}\right\|_{H^{k+4, k+4}}+\left\|n_{0}\right\|_{H^{k+3, k+4}}+\left\|n_{1}\right\|_{H^{k+2, k+4}}\right. \\
& \left.+\sum_{|\alpha| \leq k+4} \int_{0}^{t}\left\|\Gamma^{\alpha}(n u)(s)\right\| d s\right) \\
\leq & C \delta+C \sum_{|\beta|+|\gamma| \leq k+4} \int_{0}^{t}\left\|\Gamma^{\beta} n \cdot \Gamma^{\gamma} u(s)\right\| d s
\end{aligned}
$$




$$
\begin{aligned}
& \leq C \delta+C \int_{0}^{t}\left\{(1+s)^{-1+\varepsilon}|n|_{[k / 2]+2,-1}\|u\|_{k+4, \varepsilon}\right. \\
&\left.+(1+s)^{-3 / 2+2 \varepsilon}\|n\|_{k+4,0}|u|_{[k / 2]+2,-3 / 2+2 \varepsilon}\right\} d s \\
& \leq C \delta+C(1+t)^{\varepsilon} M^{2}
\end{aligned}
$$

where we have used the Sobolev inequality in the Minkowski space $\mathbf{R}^{3+1}$ as before. Hence, using the fact

$$
\left[\partial, \Gamma^{\alpha}\right]=\sum_{|\beta| \leq|\alpha|-1} C_{\beta} \Gamma^{\beta}
$$

we obtain

$$
\begin{aligned}
& (1+s)^{-\varepsilon}\left(\|\psi(t)\|_{k+4}+\|\partial \psi(t)\|_{k+4}\right) \\
\leq & C \delta+C M^{2} .
\end{aligned}
$$

We next evaluate $\varphi=\chi_{2}(u, n)$. To derive the $L^{2}$ estimate of $\varphi$, we write the solution of (2.2) as follows :

$$
\varphi=\varphi_{0}+\varphi_{1}
$$

where $\varphi_{0}$ is a solution of $\partial_{t}^{2} \varphi_{0}-\Delta \varphi_{0}=0$ with the data $n_{0}$ and $n_{1}$, and $\varphi_{1}$ is a solution of

$$
\partial_{t}^{2} \varphi_{1}-\Delta \varphi_{1}=\Delta|u|^{2}
$$

with zero data. Then, using the $L^{2}-L^{2}$ estimate by Klainerman [7], we have

$$
\begin{aligned}
\left\|\Gamma^{\alpha} \varphi_{0}(t)\right\| & \leq\left\|\Gamma^{\alpha} \varphi(0)\right\| \\
& \leq C\left(\left\|n_{0}\right\|_{H^{k+4, k+4}}+\left\|n_{1}\right\|_{H^{k+3, k+4}}\right) \\
& \leq C \delta
\end{aligned}
$$

On the other hand, to derive the $L^{2}$ estimate of $\varphi_{1}$, we rewrite (2.10) applied by $\Gamma^{\alpha}$ as the following integral equation :

$$
\Gamma^{\alpha} \varphi_{1}(t)=\sum_{|\alpha| \leq k+4} \int_{0}^{t} \frac{\sin \omega_{0}(t-s)}{\omega_{0}}\left(\partial_{s}+\partial_{x}\right) \partial \Gamma^{\alpha}\left(|u|^{2}\right)(s) d s, \quad|\alpha| \leq k+4
$$

where we have used the facts

$$
[\square, \Gamma]=0, \quad[\Gamma, \partial]=\partial
$$


Then, by integrating by parts and the Hardy-Littlewood-Sobolev inequality, we have

$$
\begin{aligned}
\left\|\Gamma^{\alpha} \varphi_{1}(t)\right\| \leq C \sum_{|\alpha| \leq k+4}\left\|\partial \Gamma^{\alpha}\left(|u|^{2}\right)(0)\right\|_{L^{6 / 5}\left(\mathbf{R}^{3}\right)} \\
\quad+C \sum_{|\alpha| \leq k+4} \int_{0}^{t}\left\|\partial \Gamma^{\alpha}\left(|u|^{2}\right)(s)\right\| d s \\
\leq C\|\partial u(0)\|_{k+4} \sum_{|\beta| \leq[(k+5) / 2]}\left\|\Gamma^{\beta} u(0)\right\|_{L^{3}\left(\mathbf{R}^{3}\right)} \\
\quad+C \int_{0}^{t}(1+s)^{-3 / 2+3 \varepsilon}|u|_{[(k+5) / 2]_{,}-3 / 2+2 \varepsilon}\|\partial u\|_{k+4, \varepsilon} d s \\
\leq C \delta^{2}+C M^{2}, \quad|\alpha| \leq k+4,
\end{aligned}
$$

since $\varepsilon<1 / 6$. Thus, from (2.11) and (2.12), we obtain

$$
\|\varphi(t)\|_{k+4} \leq C \delta+C \delta^{2}+C M^{2}
$$

Inequalities (2.8), (2.9) and (2.13) yield

$$
\|(\psi, \varphi)\|_{X} \leq C \delta+C M^{2}
$$

where $C$ does not depend on $\delta$ and $t$. If we choose $\delta>0$ and $M>0$ so that

$$
M \leq \frac{1}{4 C}, \quad \delta \leq \frac{M}{4 C}
$$

then we obtain by $(2.12)$

$$
\|(\psi, \varphi)\|_{X} \leq \frac{1}{2} M
$$

In the same way, we can prove that

$$
d(\chi(u, n), \chi(v, m)) \leq \frac{1}{2} d((u, n),(v, m)), \quad(u, n),(v, m) \in X
$$

if $\delta>0$ and $M>0$ are chosen as above. Thus, we see that if $\delta>0$ is sufficiently small, then $\chi(u, n)$ is a contraction map from $X$ into itself. Therefore, there exist unique solutions $(u, n) \in X$ of $(1.1)-(1.3)$.

Finally, the uniqueness of the solutions satisfying (1.1)-(1.3) follows by a similar argument as above. For details, see [2]. 


\section{Acknowledgements}

I would like to thank Professor Yoshi Tsutsumi for his valuable comments. I am also grateful to Professor Tohru Ozawa who pointed out mistakes in the first version of this paper.

\section{References}

[1] A. Bachelot, Problème de Cauchy globale pour des systèmes de Dirac-KleinGordon, Ann. Inst. Henri Poincaré, Physique théorique 48, 387-422 (1988).

[2] V. Georgiev, Global solution of the system of wave and Klein-Gordon equations, Math. Z. 203, 683-698 (1990).

[3] V. Georgiev, Small amplitude solutions of the Maxwell-Dirac equations, Indiana Univ. Math. J. 40, 845-883 (1991).

[4] V. Georgiev, Decay estimates for the Klein-Gordon equations, Commun. Part. Diff. Eqs. 17, 1111-1139 (1992).

[5] L. Hörmander, On Sobolev spaces associated with some Lie algebras, Report 4, 1985, Inst. Mittag-Leffler.

[6] S. Klainerman, Uniform decay estimates and the Lorentz invariance of the classical wave equations, Comm. Pure Appl. Math. 38, 321-332 (1985).

[7] S. Klainerman, The null condition and global existence to nonlinear wave equations, Lect. in Appl. Math. 23, 293-326 (1986).

[8] S. Klainerman, Remarks on the global Sobolev inequalities in the Minkowski space $R^{n+1}$, Comm. Pure Appl. Math. 40, 111-117 (1987).

[9] T. Ozawa, K. Tsutaya and Y. Tsutsumi, Normal Form and Global Solutions for the Klein-Gordon-Zakharov Equations, to appear in Ann. Inst. Henri Poincaré, Analyse non linéaire:

[10] J. Shatah, Normal forms and quadratic nonlinear Klein-Gordon equations, Comm. Pure Appl. Math. 38, 685-696 (1985). 
[11] T. Sideris, Decay estimates for the three-dimensional inhomogeneous KleinGordon equation and applications, Commun. Part. Diff. Eqs. 14, 1421-1455 (1989)

[12] J.C.H. Simon and E. Taflin, The Cauchy problem for non-linear Klein-Gordon equations, Commun. Math. Phys. 152, 433-478 (1993).

[13] W.A. Strauss, Nonlinear Wave Equations, CBMS Regional Conference Series in Mathematics, no. 73, Amer. Math. Soc., Providence, RI, 1989.

[14] S.G. Thornhill and D. ter Haar, Langmuir turbulence and modulational instability, Phys. Reports (Sect. C of Phys. Lett.) 43, 43-99 (1978). 